ORIGINAL ARTICLE

\title{
Avaliação e estabilidade da cor em filés de burriquete (Pogonias cromis) utilizando um sistema de visão computacional
}

\section{Evaluation and stability of the colour in burriquete (Pogonias cromis) fillets using a computer vision system}

\author{
Rosmer Huamán ${ }^{1}$, Giordan Fernandes da Rosa1, Carlos Prentice ${ }^{\text {1* }}$ (]) \\ ${ }^{1}$ Universidade Federal do Rio Grande (FURG), Escola de Química e Alimentos (EQA), Rio Grande/RS - Brasil \\ *Corresponding Author: Carlos Prentice, Universidade Federal do Rio Grande (FURG), Escola de Química e \\ Alimentos (EQA), Av. Itália, km 8, Carreiros, CEP: 96203-900, Rio Grande/RS - Brasil, e-mail: \\ carlos.prentice@gmail.com
}

Cite as: Huamán, R., Rosa, G. F., \& Prentice, C. (2019). Evaluation and stability of the colour in burriquete (Pogonias cromis) fillets using a computer vision system. Brazilian Journal of Food Technology, 22, e2015088. https://doi.org/10.1590/1981-6723.08815

\begin{abstract}
Resumo
A cor da superfície da carne é a primeira sensação que o consumidor percebe e utiliza como uma ferramenta para aceitar ou rejeitar um alimento. A medição e a avaliação da cor da carne podem também ser feitas por um indicador químico (metamioglobina) ou pela avaliação física, utilizando espaços da cor ( $a^{\star}$ e R). $O$ objetivo deste trabalho foi avaliar a cor e a estabilidade da cor em filés de burriquete (Pogonias cromis) utilizando parâmetros de $a^{*}$ e $\mathrm{R}$, e porcentagem de metamioglobina. Avaliaram-se a cor e a estabilidade da cor em filés de burriquete armazenados a $5 \pm 1{ }^{\circ} \mathrm{C}$ com $90 \%$ de umidade relativa, durante $57,6 \mathrm{~h}$, utilizando um sistema de visão computacional. Os parâmetros de vermelho (valor $\mathrm{a}^{*} \mathrm{e}$ R) variaram de forma inversa com a concentração de metamioglobina. A cinética foi plotada utilizando o modelo cinético, sendo que as ordens de reação para o valor $a^{*}$, R e metamioglobina foram de 2,0309; 1,2101 e 1,8324, respectivamente; a constante de reação foi de -0,000046 para o valor $a^{*}$, de -0,0013 para $\mathrm{R}$ e de 0,0002 para a metamioglobina. $O$ sistema de visão computacional se apresenta como uma interessante ferramenta para avaliar a cor e a estabilidade da cor na carne de pescado, mas também pode ser utilizado em outro tipo de produtos alimentícios.
\end{abstract}

Palavras-chave: Sistema de visão computacional; Metamioglobina; Estabilidade da cor; Pescado.

\begin{abstract}
The colour of the meat surface is the first feeling that the consumer perceives and uses as a tool to accept or reject the food. The measurement and evaluation of meat colour can be measured by a chemical indicator (metmyoglobin) or by a physical assessment using colour spaces ( $a^{*}$ and R). The objective of this study was to evaluate the colour and colour stability in burriquete (Pogonias cromis) fillets using the red parameters ( $a^{*}$ value and $\mathrm{R}$ ) and chemical analysis (\% of metmyoglobin). The colour and colour stability of burriquete fillets stored at $5 \pm 1{ }^{\circ} \mathrm{C}$ with $90 \%$ of relative humidity for $57.6 \mathrm{~h}$ was evaluated using a computer vision system. The red parameters ( $\mathrm{a}^{*}$ value and $\mathrm{R}$ ) varied inversely with the metmyoglobin concentration. The kinetics were plotted using the kinetic model.
\end{abstract}


The reaction orders for the $a^{*}$ value, $R$ and metmyoglobin were $2.0309,1.2101$ and 1.8324 , respectively and the reaction constants were -0.000046 for the $a^{*}$ value, -0.0013 for $R$ and 0.0002 for metmyoglobin. The computer vision system was shown to be a powerful tool for assessing the colour and colour stability in fish meat but could also be used in other types of food product.

Keywords: Computer vision system; Metmyoglobin; Colour stability; Fish.

\section{Introdução}

Os consumidores utilizam a cor da superfície da carne como um indicador da salubridade. A mioglobina é o maior contribuinte da cor do músculo, dependendo do estado redox (desoximioglobina, oximioglobina e metamioglobina) e da concentração (Faustman \& Cassens, 1990). As proteínas heme, mioglobina e hemoglobina são os maiores pigmentos encontrados em pescado, especialmente em músculo escuro. A mioglobina é a proteína heme responsável pela cor da carne. A oxidação do átomo central de ferro dentro do grupo heme é responsável pela descoloração, uma variação de oximioglobina (vermelho) a metamioglobina (cinzento). Quando o ferro heme ferroso oxida em forma férrica, o oxigênio é liberado e substituído por uma molécula de água (Faustman et al., 2010).

A medição física da cor da carne, utilizando espaços da cor, ou a química, mediante um indicador (metamioglobina), é útil para avaliar a estabilidade da cor da carne. O método do sistema de visão computacional fornece medições validadas que reproduzem a cor real do alimento (Girolami et al., 2013). Nos sistemas de visão computacional, as imagens são adquiridas por uma máquina fotográfica e salvas em um espaço tridimensional de cor RGB (vermelho, verde, azul) (Du \& Sun, 2005). Esses três espaços são suficientes para descrever a sensação da cor (Sharifzadeh et al., 2014). Deste espaço de cor, pode-se utilizar o valor R para avaliar a cor vermelha da carne. Usualmente, a cor dos alimentos se mede no espaço da cor L*a*b* (León et al., 2006). Os instrumentos eletrônicos usados para medir a cor definem a cor em termos de $a^{*}$ (vermelho/verde), b* (amarelo/azul) e L* (luminosidade) (Yagiz et al., 2009). Do espaço L*a*b*, o valor $a^{*}$ pode ser utilizado para avaliar a cor vermelha da carne.

Devido à sua sensibilidade e ao baixo custo, o sistema de visão computacional pode ser utilizado como uma ferramenta prática para detectar as mudanças na cor da carne (Larraín et al., 2008). Quevedo et al. (2013) avaliaram a cor e a estabilidade da cor em carne de porco utilizando um sistema de visão computacional. A relação entre a medição instrumental da cor e a estabilidade da cor da carne com a mioglobina e suas interações com multiplex fatores extrínsecos e intrínsecos da carne é importante porque estes governam a cor da carne.

Neste trabalho, o objetivo foi avaliar a cor e a estabilidade da cor em filés de burriquete (Pogonias cromis) utilizando parâmetros de vermelho (valor $\mathrm{a}^{*} \mathrm{e}$ R) e a análise química (porcentagem de metamioglobina).

\section{Material e métodos}

\subsection{Sistema de visão computacional}

O sistema de visão computacional utilizado nesta pesquisa estava composto de sistema de aquisição de imagens (sistema de iluminação e máquina fotográfica), um laptop e software para processar as imagens. As imagens usadas neste trabalho foram obtidas com um sistema de aquisição de imagens (Figura 1), construído no Laboratório de Tecnologia de Alimentos da Universidade Federal do Rio Grande. A qualidade das imagens capturadas depende das condições da iluminação (Dowlati et al., 2013). Quatro lâmpadas fluorescentes de 18W/D65 (Taschibra, Brasil) - com uma temperatura de cor de $6.400 \mathrm{~K}$, um índice de reprodução da cor de 70\% e um fluxo luminoso de $900 \mathrm{~lm}$ - foram utilizadas para obter imagens com alta 
qualidade sob condições de iluminação reprodutíveis (Dowlati et al., 2013). As lâmpadas com $60 \mathrm{~cm}$ de comprimento foram instaladas na forma de um quadrado a $35 \mathrm{~cm}$ de altura sobre a amostra, num ângulo de $45^{\circ}$.

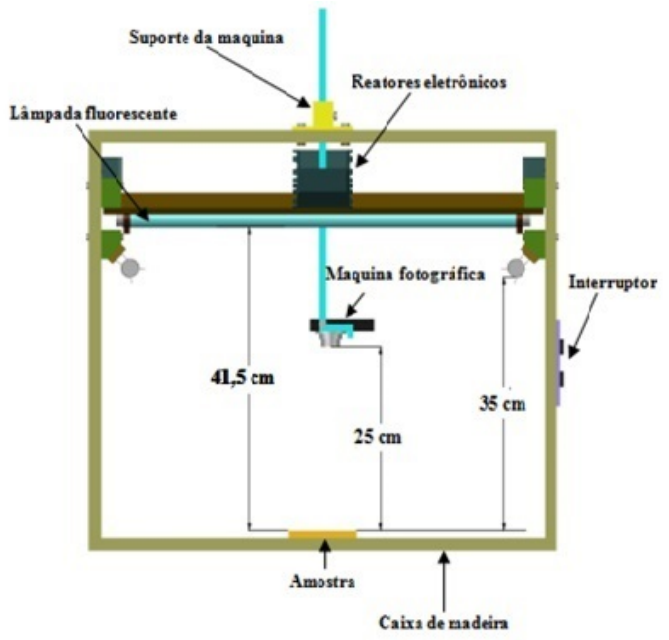

Figura 1. Vista frontal do sistema de aquisição de imagens adaptado de León et al. (2006).

A fonte de luz padrão foi a D65, que é comumente utilizada na pesquisa com alimentos (Quevedo et al., 2010). O sistema de aquisição de imagens foi construído de madeira, com um tamanho de $71,5 \mathrm{~cm} \times 69,1 \mathrm{~cm}$ $\times 58,8 \mathrm{~cm}$. Para minimizar a luz do fundo e a reflexão, as paredes internas foram pintadas de cor preta (León et al., 2006; Dowlati et al., 2013; Quevedo et al., 2010). As amostras foram situadas numa bandeja que se encontrava fixa no centro do sistema. Uma máquina fotográfica da marca Sony modelo CCD Power Shot (14,1 megapixels) foi empregada para adquirir as imagens das amostras e, a distância vertical entre a lente da máquina fotográfica e as amostras foi de $25 \mathrm{~cm}$. A máquina fotográfica foi conectada a um laptop (Intel-Pentium 7, 2,00 GHZ de processador, 8,00 GB RAM, $500 \mathrm{~GB}$ de disco duro) via interface USB. As imagens foram adquiridas em sua máxima resolução $(4.320 \times 3.240$ pixels $)$ e salvas no formato TIFF. Todas as imagens foram capturadas com características constantes da máquina fotográfica como modo de operação manual, flash e Zoom desligado, velocidade ISSO 80, ajuste de exposição 0, apertura AV f/2,7 e distância focal $35 \mathrm{~mm}$.

Para calibrar o sistema de visão computacional, foram criadas 60 cartas de cor (Figura 2) no programa Adobe Photoshop cc. As cartas de cor foram impressas em papel fotográfico profissional fosco de Fujifilm. De cada carta de cor, foram medidos os valores $\mathrm{L}^{*}$, $\mathrm{a}^{*}$ e b* utilizando um colorímetro Konica Minolta CR-400 e, adicionalmente, tomou-se uma imagem digital sRGB, sendo os valores de cor R, G e B de cada carta medidos usando o programa MATLAB ${ }^{\circledR} \mathrm{R} 2012 \mathrm{a}$ (The Mathwords Company).

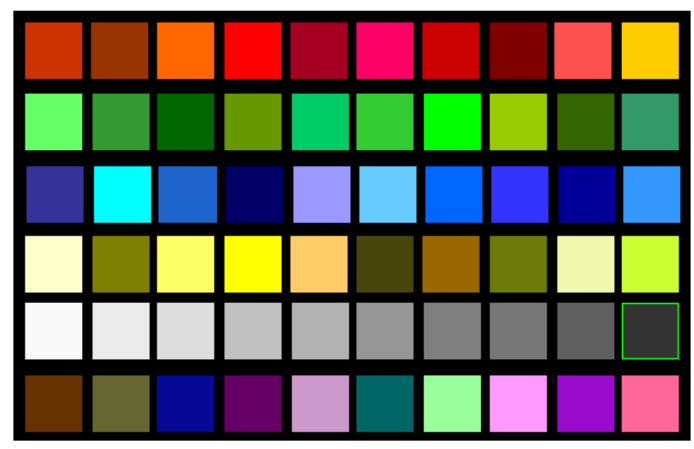

Figura 2. Cartas de cor utilizadas no processo de calibração do sistema de visão computacional. 
A metodologia desenvolvida por León et al. (2006) foi utilizada para calibrar o sistema de visão computacional.

$$
\left[\begin{array}{l}
L^{*} \\
a^{*} \\
b^{*}
\end{array}\right]=\left[\begin{array}{l}
\theta_{11} \theta_{12} \theta_{13} \theta_{14} \theta_{15} \theta_{16} \theta_{17} \theta_{18} \theta_{19} \theta_{1,10} \\
\theta_{21} \theta_{22} \theta_{23} \theta_{24} \theta_{25} \theta_{26} \theta_{27} \theta_{28} \theta_{29} \theta_{2,10} \\
\theta_{31} \theta_{32} \theta_{13} \theta_{34} \theta_{35} \theta_{36} \theta_{37} \theta_{38} \theta_{39} \theta_{3,10}
\end{array}\right]\left[\begin{array}{l}
R \\
G \\
B \\
R G \\
R B \\
G B \\
R^{2} \\
G^{2} \\
B^{2} \\
1
\end{array}\right]
$$

Na Equação 1, os valores $L * a * b *$ são os estimativos dos valores $L^{*} a * b *$ originais aferidos pelo colorímetro Konica Minolta CR-400 nas cartas da cor e os $\theta$ 's são os coeficientes do modelo quadrático. O erro meio normalizado (E), na estimativa de cada uma das variáveis $L^{*}, a^{*}$ e $b^{*}$, foi obtido por comparação das medições $\left(\mathrm{L}^{*} \mathrm{a} \mathrm{b}^{*}\right)$ do colorímetro Konica Minolta CR-400 com as estimações ( $\mathrm{L}^{*} \mathrm{a}^{*} \mathrm{~b} *$ ) pelo modelo.

$$
\boldsymbol{E}_{\boldsymbol{X}}=\frac{1}{\boldsymbol{N} . \Delta \boldsymbol{X}} \cdot \sum\left|\boldsymbol{X}_{0, i}-\boldsymbol{X}_{e, i}\right|
$$

Na Equação 2, $N$ é o número de dados experimentais $(i)$ e, $X$ a variável correspondente ( $\mathrm{L}^{*}, \mathrm{a}^{*}$ ou $\left.\mathrm{b}^{*}\right)$; subíndice $o$ é a variável original; subíndice $e$ é a variável estimada, $\Delta X$ é o rango de cada variável, $\Delta L^{*}=100$, $\Delta a^{*}=120, \Delta b=120$, respectivamente. O erro geral do modelo foi calculado utilizando a Equação 3.

$\overline{\boldsymbol{e}}=\frac{\boldsymbol{e}_{L}+\boldsymbol{e}_{a}+\boldsymbol{e}_{b}}{3}$

Na Equação 3, $\bar{e}$ é o erro geral do modelo, $e_{L}, e_{a}$ e $e_{b}$ são os erros para L*, a* e b* calculados com a Equação 2. Os coeficientes $\theta$ na Equação 1 foram estabilizados minimizando o erro da função (Equação 2). A minimização foi realizada num algoritmo escrito em MATLAB ${ }^{\circledR} \mathrm{R} 2012 \mathrm{a}$ (The Mathwords Company)

\subsection{Preparação da amostra}

Os espécimes de burriquete foram obtidos de um provedor local (Associação de Pescadores da Vila São Miguel- APESMI, Rio Grande, Brasil) após dois dias da captura em alto mar e foram transportados para o Laboratório de Tecnologia de Alimentos da Universidade Federal do Rio Grande, numa caixa de isopor com gelo. No Laboratório, os pescados foram filetados e, de cada pescado, obtiveram-se dois filés; em seguida, de cada filé, seis porções $(3 \mathrm{~cm} \times 3 \mathrm{~cm} \times 1 \mathrm{~cm})$ foram obtidas (ao todo, foram 12 porções para cada pescado) e colocadas num refrigerador de bancada, para avaliar a cor utilizando um sistema de visão computacional. Além disso, 24 amostras também foram colocadas no refrigerador de bancada, para as análises químicas.

\subsection{Análise das imagens}

Um sistema de visão computacional, descrito na seção 2.1, foi utilizado para capturar as imagens (espaço de cor sRGB, em sua máxima resolução). Uma metodologia descrita por Quevedo et al. (2013) foi utilizada para analisar as imagens. As imagens foram capturadas em quintuplicada a zero; 9,6;16,8;24; 33,6;40,8; 48 e 57,6 horas, respectivamente. Um total de 40 imagens foi processado para cada amostra (porção do pescado) 
durante o armazenamento. As imagens usadas estavam no espaço da cor sRGB e foram transformadas no espaço da cor CIEL*a*b*, utilizando um modelo quadrático desenvolvido por León et al. (2006). Esta transformação considera a influência das variáveis quadráticas (RGB) na estimativa dos valores CIEL*a*b*, os erros na estimativa para $L^{*}, a^{*} y b^{*}$ que foram de $1,41 \% ; 1,66 \% ; 2,08 \%$ e um erro geral de $1,72 \%$ para $o$ modelo. No espaço da cor sRGB, o parâmetro vermelho (R) foi utilizado diretamente para registrar as mudanças na intensidade do vermelho; no espaço de cor CIEL*a*b*, foi utilizado o valor cromático a*. As intensidades das superfícies foram geradas (correspondentes a $\mathrm{R}$ e $\mathrm{a}^{*}$ ) e usadas para descrever as mudanças na intensidade de vermelho na superfície da imagem da amostra. Foi obtida a intensidade da superfície (SI) de uma imagem da superfície da amostra plotando as coordenadas dos pixels (X, Y) contra os valores de intensidade no eixo Z. Esta pode ser definida como um mapa da intensidade da cor que mostra a distribuição da intensidade da cor na imagem da amostra. Um algoritmo escrito no Programa MATLAB ${ }^{\circledR}$ R2012a (The Mathwords Company) foi utilizado para obter os gráficos de intensidade das superfícies.

$\mathrm{O}$ algoritmo escrito no MATLAB para gerar a superfície de intensidade das amostras analisadas constava dos seguintes passos:

- Capturou-se uma imagem da amostra;

- Carregou-se a imagem ao MATLAB;

- Segmentou-se a parte da amostra a ser analisada $(3 \mathrm{~cm} \times 3 \mathrm{~cm}$ ou 290 pixels $\times 290$ pixels $)$;

- Extraiu-se o valor R da imagem segmentada no espaço sRGB;

- Conversão do espaço sRGB da imagem selecionada ao espaço CIEL*a*b* utilizando os parâmetros do modelo quadrático obtidos no processo de calibração do sistema de visão computacional;

- Extraiu-se o valor cromático a* do espaço CIEL*a*b*;

- A superfície de intensidade foi obtida plotando as coordenadas dos pixels (X, Y) contra os valores de intensidade no eixo Z.

\subsection{Conteúdo de metamioglobina}

A metodologia descrita por Quevedo et al. (2013) foi utilizada para determinar o conteúdo de metamioglobina. Três amostras foram removidas das 24 colocadas no refrigerador de bancada, a cada momento da análise das imagens. As amostras foram homogeneizadas juntas; assim, cinco gramas de carne homogeneizada manualmente foram colocados num béquer de $100 \mathrm{~mL}$ e foram adicionados $25 \mathrm{~mL}$ de uma solução buffer fosfato $(\mathrm{pH} \mathrm{6,8)}$. A mistura foi homogeneizada por $10 \mathrm{~s}$. As amostras homogeneizadas foram depositadas em tubos de polipropileno de $500 \mathrm{~mL}$ e colocadas num refrigerador de bancada por uma hora, a $5 \pm 1{ }^{\circ} \mathrm{C}$, e depois centrifugadas a $5.000 \mathrm{rpm}$ por $30 \mathrm{~min}$ a $4{ }^{\circ} \mathrm{C}$; de cada amostra o sobrenadante foi filtrado em papel de filtro Whatman $\mathrm{N}^{\circ} 1$ e a absorbância (A) foi lida em triplicata para 700, 572 e $525 \mathrm{~nm}$, utilizando um espectrofotômetro Kasuaki (Modelo IL - 592). A porcentagem da metamioglobina foi determinada usando a Equação 4 (Quevedo et al., 2013):

MetMb $=\left\{1.395-\left[\frac{\left(\boldsymbol{A}_{572}-\boldsymbol{A}_{700}\right)}{\left(\boldsymbol{A}_{525}-\boldsymbol{A}_{700}\right)}\right]\right\} * 100$

\subsection{Modelo cinético da oxidação para o valor cromático a*, vermelho (R) e conteúdo de metamioglobina (\%)}

É uma ordem para expressar a proporção da cinética de escurecimento, o modelo cinético, descrito por Quevedo et al. (2013). Foi aplicado aos dados, seguindo a Equação 5: 


$$
\frac{\mathbf{C}_{\mathbf{t}}}{\mathbf{C}_{0}}=\exp \left(\mathbf{k}^{*} \mathbf{t}^{\mathrm{n}}\right)
$$

Na Equação 5, $C_{t}$ é o valor médio de R, o valor médio de a* e a concentração de metamioglobina (\%) para o tempo $t$, respectivamente; Co é o valor inicial $\mathrm{R}$, o valor inicial de $\mathrm{a}^{*} \mathrm{e}$ a concentração inicial de metamioglobina (\%). K é a constante de velocidade de reação e " $n$ " é um parâmetro chamado "fator de forma". O fator de forma, na Equação, é uma medição do grau de concavidade e a direção da curva plotada (Peleg et al., 2012). Os dados foram ajustados ao modelo pelo método dos mínimos quadrados, usando a função solver, de Excel 2010 (Microsoft ${ }^{\circledR}$ Company).

\section{Resultados e discussão}

A cor da carne fresca é uma importante propriedade, que influencia no valor do mercado e na decisão de compra do consumidor (Tan, 2004; Quevedo et al., 2013). A visão computacional tem a habilidade para medir a cor com elevada resolução espacial e um alto desempenho na gravação e avaliação de pequenas mudanças na cor dos alimentos (Oliveira \& Balaban, 2006). A superfície de intensidade (baseado em a*) da amostra para o início é muito irregular (Figura 3) e estas irregularidades ocorrem porque a cor não é uniforme na superfície da amostra.
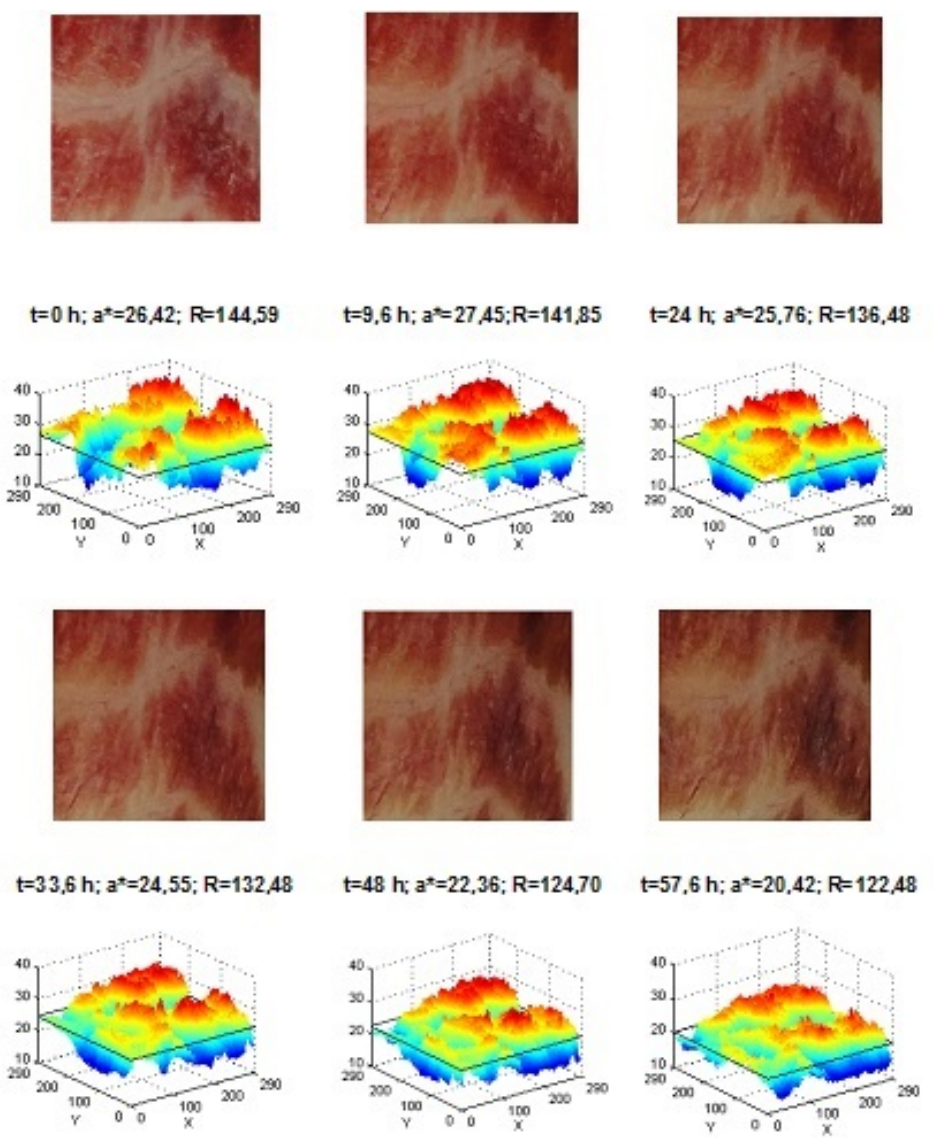

Figura 3. Amostras de filé de burriquete armazenado a uma temperatura de $5^{\circ} \pm 1 \mathrm{C}$ ), apresentando a superfície de intensidade para o valor a* e seus respectivos valores cromáticos a* e R, durante 57,6 h de armazenamento. 
Segundo a Figura 3, a forma geométrica da superfície da intensidade para a amostra durante o tempo de exposição ao ar mostra que a oxidação da mioglobina a metamioglobina se realiza de uma forma não homogênea. A superfície de intensidade para $\mathrm{R}$ foi análoga ao valor cromático $\mathrm{a}^{*}$. Este comportamento se deve provavelmente a mudanças no conteúdo de mioglobina e hemoglobina, que são responsáveis pela cor do pescado fresco (Yarnpakdee et al., 2012). A concentração da mioglobina no tecido muscular pode mudar devido a vários fatores, incluindo tipo de fibra, atividade muscular e idade do animal (Haard, 1992).

A avaliação instrumental da cor representa uma alternativa eficiente aos procedimentos tradicionais de extração de pigmentos, em estudos de controle, em que a quantidade de pigmentos não é requerida (Olsen \& Mortensen, 1997). O sistema de visão computacional pode ser utilizado para avaliar a descoloração da carne (Larraín et al., 2008). Os valores médios de a* e R decrescem com o tempo (Figuras 4 e 5), exceto para períodos curtos de armazenamento (9,6 horas), nos quais a cor vermelha $\left(\mathrm{a}^{*}\right)$ aumentou levemente. Um pequeno aumento da cor vermelha acontece quando a carne cortada é exposta ao ar por curto tempo (Kannan et al., 2001).

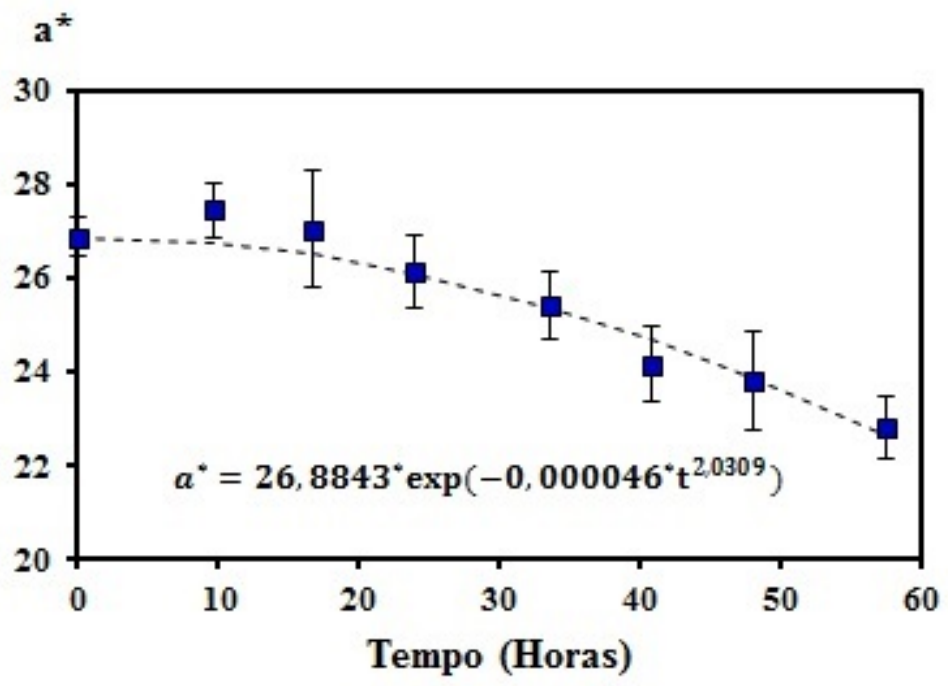

Figura 4. Intensidade das mudanças de vermelho, utilizando os valores médios de a* na superfície da imagem (tomaram-se em conta todas as amostras analisadas).

Esse aumento da cor vermelha é devido à exposição da carne fresca ao ar por períodos curtos, já que a mioglobina se combina com o oxigênio para formar a oximioglobina, que apresenta cor vermelha brilhante. A oximioglobina reflete mais luz que a mioglobina (Brewer, 2004), o que explica o aumento do valor cromático $\mathrm{a}^{*}$. Chaijan et al. (2005) associaram o escurecimento do músculo de sardinha e cavala com a oxidação da mioglobina. A diminuição do valor a* e $\mathrm{R}$ em tempos prolongados é devida à descoloração da superfície da carne do pescado e, com frequência, é referida a quantidade de área superficial coberta de metamioglobina (Mancini \& Hunt, 2005). 


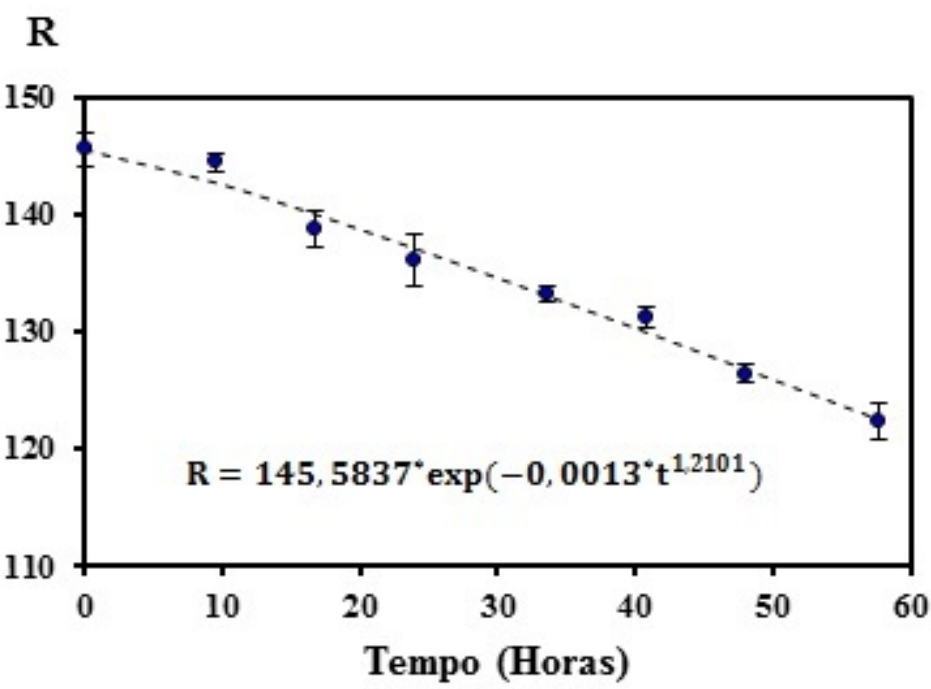

Figura 5. Intensidade das mudanças de vermelho, utilizando os valores médios de vermelho (R) na superfície da imagem (considerando-se todas as amostras analisadas).

Uma ordem de reação ( $>1)$ foi estabelecida quando os valores de a* e R foram utilizados (os valores de $n$ foram de 2,0309 e 1,2101, respectivamente). Quevedo et al. (2013) calcularam um valor similar para a* $(2,2)$ e um valor para $\mathrm{R}(4,8)$, quando plotaram a diminuição dos valores de $\mathrm{a} * \mathrm{e} \mathrm{R}$ durante o tempo de estabilização da cor em carne de porco, e a razão da cinética baseada nos valores de $\mathrm{a}^{*}$ e $\mathrm{R}$, com um decréscimo de 0,0011 unidade de $\mathrm{R}$ em média por minuto, com um decréscimo de 0,17 unidade de a* por minuto. As taxas da cinética baseadas nos valores de $a^{*}$ e $R$, neste estudo, mostraram um decréscimo de 0,000046 unidade de $\mathrm{a}^{*}$ em média, por hora, e um decréscimo de 0,0013 unidade de $\mathrm{R}$ em média, por hora. Estas diferenças na ordem de reação e a razão da cinética se devem aos diferentes tipos de matéria-prima utilizada.

A concentração de metamioglobina aumentou com o tempo (Figura 5), como reportado por Chaijan et al. (2008).

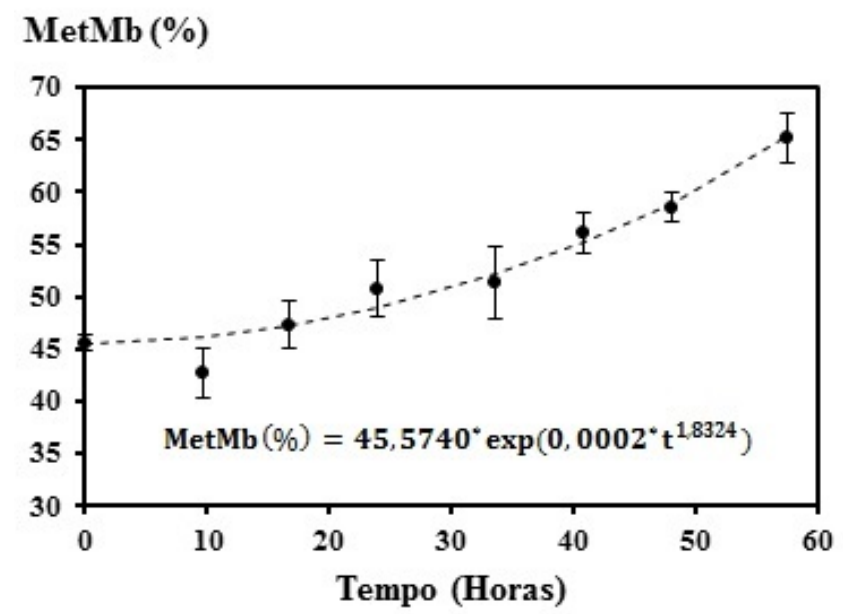

Figura 6. Mudanças na concentração de metamioglobina (\%) em filés de burriquete, durante o tempo (57,6 h).

Em carne crua, existe um ciclo dinâmico na presença de oxigênio dos três pigmentos mioglobina, oximioglobina e metamioglobina, e são constantemente interconvertidas, em que as três formas estão em equilíbrio uma com outra. Na Figura 6, pode-se observar que, em tempos curtos, a concentração de metamioglobina decresce e isto é porque a mioglobina se une covalentemente com o oxigênio, produzindo a 
oximioglobina (Brewer, 2004); já em tempos mais prolongados, a concentração de metamioglobina aumenta como consequência da mudança da oximioglobina a metamioglobina, quando é mais difícil que os três pigmentos se encontrem em equilíbrio, porque a metamioglobina é mais estável que a oximioglobina (Thiansilakul et al., 2011). A taxa da mudança do conteúdo de metamioglobina foi de 0,0002 unidade por hora, durante um período de 57,6 horas. A ordem de reação $(>1)$ foi estabelecida na oxidação da metamioglobina em filés de burriquete (Pogonias cromis) e resultados similares foram reportados pelo Quevedo et al. (2013), durante a avaliação da estabilização da cor em carne de porco.

\section{Conclusões}

Os parâmetros de vermelho (valores a* e R) e a análise química foram utilizados para avaliar a cor e a estabilidade da cor em filés de burriquete (Pogonias cromis). Os parâmetros de vermelho (valores a* e R) evidenciaram uma relação inversa com as mudanças na concentração de metamioglobina. Os parâmetros de vermelho (valores a* e R) aumentaram no início ( 0 a 9,6 horas) e depois decresceram com o tempo de armazenamento, o que estava de acordo com um escurecimento na superfície da amostra. A concentração da metamioglobina aumentou na amostra, evidenciando o progresso da oxidação da mioglobina a metamioglobina. Os parâmetros de vermelho (valor a* e R) e a análise química são úteis para avaliar a cor e a estabilidade da cor da carne.

\section{Referências}

Brewer, S. (2004). Irradiation effects on meat color: A review. Meat Science, 68(1), 1-17. PMid:22062002. http://dx.doi.org/10.1016/j.meatsci.2004.02.007

Chaijan, M., Benjakul, S., Visessanguan, W., \& Faustman, C. (2005). Changes of pigments and color in sardine (Sardinella gibbosa) and mackerel (Rastrelliger kanagurta) muscle during iced storage. Food Chemistry, 93(4), 607-617. http://dx.doi.org/10.1016/j.foodchem.2004.10.035

Chaijan, M., Benjakul, S., Visessanguan, W., Lee, S., \& Faustman, C. (2008). Interaction of fish myoglobin and myofibrillar proteins. Journal of Food Science, 73(5), 292-298. PMid:18576972. http://dx.doi.org/10.1111/j.1750-3841.2008.00749.x

Dowlati, M., Mohtasebi, S. S., Omid, M., Razavi, S. H., Jamzad, M., \& De la Guardia, M. (2013). Freshness assessment of gilthead sea bream (Sparus aurata) by machine vision based on gill and eye color changes. Journal of Food Engineering, 119(2), 277-287. http://dx.doi.org/10.1016/j.jfoodeng.2013.05.023

Du, C.-J., \& Sun, D.-W. (2005). Comparison of three methods for classification of pizza topping using different colour space transformations. Journal of Food Engineering, 68(3), 277-287. http://dx.doi.org/10.1016/j.jfoodeng.2004.05.044

Faustman, C., \& Cassens, R. G. (1990). The biochemical basis for discoloration in fresh meat: A review. Journal of Muscle Foods, 1(3), 217-243. http://dx.doi.org/10.1111/j.1745-4573.1990.tb00366.x

Faustman, C., Sun, Q., Mancini, R., \& Suman, S. P. (2010). Myoglobin and lipid oxidation interactions: Mechanistic bases and control. Meat Science, 86(1), 86-94. PMid:20554121. http://dx.doi.org/10.1016/j.meatsci.2010.04.025

Girolami, A., Napolitano, F., Faraone, D., \& Braghieri, A. (2013). Measurement of meat color using a computer vision system. Meat Science, 93(1), 111-118. PMid:22981646. http://dx.doi.org/10.1016/j.meatsci.2012.08.010

Haard, N. F. (1992). Biochemistry and chemistry of color and color change in seafoods. In G. J. Flick \& R. E. Martin (Eds.), Advances in seafood biochemistry (pp. 312-319). Louisiana: Technomic Pub.

Kannan, G., Kouakou, B., \& Gelaye, S. (2001). Color changes reflecting myoglobin and lipid oxidation in chevon cuts during refrigerated display. Small Ruminant Research, 42(1), 67-74. PMid:11163717. http://dx.doi.org/10.1016/S0921-4488(01)002322

Larraín, R., Schaefer, D. M., \& Reed, J. D. (2008). Use of digital images to estimate CIE color coordinates of beef. Food Research International, 41(4), 380-385.

León, K., Mery, D., Pedreschi, F., \& León, J. (2006). Color measurement in L*a*b* units from RGB digital images. Food Research International, 39(10), 1084-1091.

Mancini, R. A., \& Hunt, M. C. (2005). Current research in meat color. Meat Science, 71(1), 100-121. PMid:22064056. http://dx.doi.org/10.1016/j.meatsci.2005.03.003

Oliveira, A. C. M., \& Balaban, M. O. (2006). Comparison of a colorimeter with a machine vision system in measuring color of Gulf of Mexico sturgeon fillets. Applied Engineering in Agriculture, 22(4), 583-587. http://dx.doi.org/10.13031/2013.21211

Olsen, R., \& Mortensen, A. (1997). The influence of dietary astaxanthin and temperature on flesh colour in Arctic charr Salvelinus alpinus L. Aquaculture Research, 28(1), 51-58. http://dx.doi.org/10.1111/j.1365-2109.1997.tb01314.x 
Avaliação e estabilidade da cor em filés de burriquete (Pogonias cromis) utilizando um sistema de visão computacional Huamán, R. et al.

Peleg, M., Normand, M., \& Corradini, M. (2012). The Arrhenius equation revisited. Critical Reviews in Food Science and Nutrition, 52(9), 830-851. PMid:22698273. http://dx.doi.org/10.1080/10408398.2012.667460

Quevedo, R., Aguilera, J. M., \& Pedreschi, F. (2010). Color of salmon fillets by computer vision and sensory panel. Food and Bioprocess Technology, 3(5), 637-643. http://dx.doi.org/10.1007/s11947-008-0106-6

Quevedo, R., Valencia, E., Cuevas, G., Ronceros, B., Pedreschi, F., \& Bastías, J. M. (2013). Color changes in the surface of fresh cut meat: A fractal kinetic application. Food Research International, 54(2), 1430-1436. http://dx.doi.org/10.1016/j.foodres.2013.10.006

Sharifzadeh, S., Clemmensen, L., Borggaard, C., Støier, S., \& Ersbøll, B. K. (2014). Supervised feature selection for linear and non-linear regression of $\mathrm{L}^{*} \mathrm{a}^{*} \mathrm{~b}^{*}$ color from multispectral images of meat. Engineering Applications of Artificial Intelligence, 27, 211-227. http://dx.doi.org/10.1016/j.engappai.2013.09.004

Tan, J. (2004). Meat quality evaluation by computer vision. Journal of Food Engineering, 61(1), 27-34. http://dx.doi.org/10.1016/S0260-8774(03)00185-7

Thiansilakul, Y., Benjakul, S., \& Richards, M. (2011). Isolation, characterisation and stability of myoglobin from Eastern little tuna (Euthynnus affinis) dark muscle. Food Chemistry, 124(1), 254-261. PMid:25214357.

http://dx.doi.org/10.1016/j.foodchem.2010.06.028

Yagiz, Y., Balaban, M., Kristinsson, H. G., Welt, B. A., \& Marshall, M. R. (2009). Comparison of Minolta colorimeter and machine vision system in measuring colour of irradiated Atlantic salmon. Journal of the Science of Food and Agriculture, 84(4), 728-730. http://dx.doi.org/10.1002/jsfa.3467

Yarnpakdee, S., Benjakul, S., \& Kristinsson, H. (2012). Effect of pretreatments on chemical compositions of mince from Nile tilapia (Oreochromis niloticus) and fishy odor development in protein hydrolysate. International Aquatic Research., 4(7), 1-16.

Financiamento: Organização dos Estados Americanos (OEA). 